\title{
基于阶比跟踪的带钢冷轧过程轧辊偏心信号分析
}

\author{
刘 涛 ${ }^{1,2}$ 崔会宝 ${ }^{1,2}$ 陈 刚 1,2 \\ (1. 燕山大学河北省重型机械流体动力传输与控制重点实验室 秦皇岛 066004;
}

2. 先进锻压成形技术与科学教育部重点实验室(燕山大学) 秦皇岛 066004)

\begin{abstract}
摘要: 针对板带轧制厚度控制过程中轧辊偏心扰动难以在线测量和准确表达问题, 提出新的系统解决方法。根据板带轧制原 理, 建立轧辊偏心在线软测量模型, 通过采集得到的压下缸位移、轧制力、出口板厚等数据对轧制过程中的轧辊偏心信号进 行计算, 从而解决车辊偏心难以在线测量的问题。为解决轧制过程速度变化带来的偏心信号频率和周期的变化问题, 在轧辊 偏心分析中, 引入阶比跟踪技术计算角域等角度采样时标, 通过拉格朗日插值计算实现角域重采样, 把轧制过程数据的时间 序列转化为轧辊转动等角度采样的角域序列。同时, 通过厚度信号的角域平移, 解决由板厚信号滞后带来的数据不匹配问题。 利用小波滤波、相干时间平均法实现依据带钢冷轧过程数据进行轧辊角域偏心波形的分析和提取, 为实现轧辊偏心的有效补 偿打下基础。
\end{abstract}

关键词：阶比分析；轧辊偏心；冷带轧机；相干平均

中图分类号: TG331

\section{Analysis of Roll Eccentricity Signal in Strip Cold Rolling Process Based on Order Tracking}

\author{
LIU Tao ${ }^{1,2}$ CUI Huibao ${ }^{1,2}$ CHEN Gang ${ }^{1,2}$
}

(1. Hebei Province Key Laboratory of Heavy Machinery Fluid Power Transmission and Control, Yanshan University, Qinhuangdao 066004;

2. Key Laboratory of Advanced Forging \& Stamping Technology and Science(Yanshan University), Ministry of Education of China, Qinhuangdao 066004)

\begin{abstract}
Aim at the problem of roll eccentricity in measuring on line and presenting accuracy, an new effective solution is provided. According to the theory of plate and strip rolling, the roll eccentricity online soft measurement model is established, through the collected pressing cylinder displacement, rolling force, export plate thickness data during the rolling process, the roll eccentricity can be estimated. In order to solve the roll eccentricity signal frequency and cycle diversification when the rolling speed changes, the order tracking technique is applied, the time marks of same angle sampling are calculated, and the values of resample in angle domain are calculated by Lagrange interpolation method. The time sequence of rolling process data is translated into angle domain sequence. And the mismatching of data due to the lag of thickness signal is solved by moving in angle domain. The signal of roll eccentricity is analysis and extracted by wavelet filtering and coherence time average method, which is the basement work to compensate the roll eccentricity effectively.
\end{abstract}

Key words: order tracing; roll eccentricity; cold strip mill; coherent average

\section{0 前言}

在带钢冷轧过程中, 当板带厚度较薄、厚度精 度要求较高时, 消除轧辊偏心对板厚的影响变得非 常重要。轧辊偏心是由于轧辊和轧辊轴承形状的不

20140507 收到初稿, 20141110 收到修改稿
规则引起的。这些不规则可能来自设计、制造、修 磨、装配、磨损、热变形等过程, 包括轧辊本身的 非圆性, 以及轧辊几何轴心与其旋转轴心的不重合。 目前解决轧辊偏心的方法主要包括预防控制、被动 控制以及主动补偿三种方法 ${ }^{[1-2]}$ 。其中主动补偿的方 法是依据测量、分析得到的偏心信号, 在辊缝位置 控制中加以针对性的补偿。这种方法不受制造水平 的制约, 理论上可以消除轧辊偏心的大部分影响, 
是目前主要的研究方向, 并取得了一些研究成果。 但是通常是在 “空压靠” 过程中对偏心信号进行提 取, 难以避免偏心信息不可靠, 准确性不高的问题。

在轧制过程中, 轧辊间会发生打滑, 造成偏心 信号的波形特征改变, 因此依靠 “空压靠” 下提取 的偏心信号进行补偿会造成误差。只有在轧制过程 中对轧辊偏心信号进行分析, 并不断地进行校正, 偏心的补偿才更具工程意义。然而在轧制过程中, 轧制速度和轧辊的转速会发生变化, 因此在轧制过 程中, 轧辊偏心信号虽具有一定的周期性, 但本质 上属于非平稳信号, 如果应用常规时频分析法对偏 心信号进行提取, 必然会导致偏心信号波形的不稳 和频率的混叠, 产生较大的误差。

阶比分析源自旋转机械振动信号分析领域, 其 实质是针对非平稳振动信号, 通过重采样技术把时 域的等周期采样信号转换成角域采样信号, 然后再 进行角频变换, 分析振动信号的频域特征。阶比分 析可有效地消除转速变化造成的振动频率变化, 以 及由此带来的频率混叠问题 ${ }^{[3-5]}$, 其关键步骤, 即采 集数据的时角域转换过程, 称为阶比跟踪。将阶比 跟踪技术应用到轧辊偏心信号分析中, 可以解决轧 制速度变化带来的轧辊偏心分析中的难题, 使得基 于带钢冷轧过程数据的轧辊偏心分析成为可能。

\section{1 轧辊偏心在线软测量模型}

以四辊轧机为例, 压下系统的工作原理见图 1。

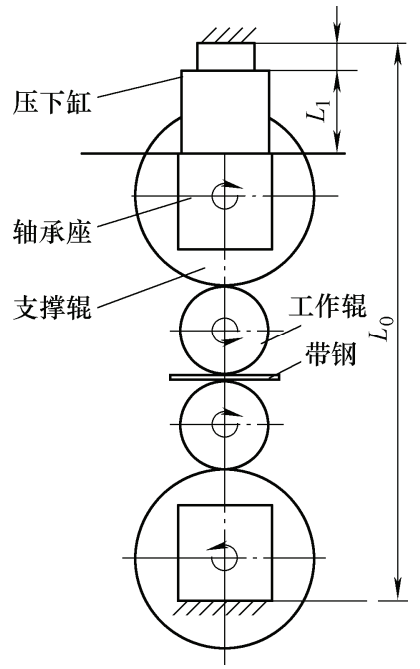

图 1 轧机压下系统原理图

轧机的工作部分主要包括上下两个支承辊和 两个工作辊。电动机通过齿轮箱带动上下的工作辊 转动, 支承辊依靠与工作辊的紧密接触而产生的摩 擦力转动。各轧辊坚直方向的位置决定了空载辊缝 的大小，上支撑辊轴承座与液压缸的缸体通过球面
垫相连, 它的坚直位置由液压缸的活塞杆位移决定, 下支撑辊轴承座固定，而工作辊辊面与支承辊面接 触, 在坚直方向完全由支承辊定位。轧制过程中, 自动板厚控制系统控制信号通过电液伺服阀控制压 下缸产生活塞位移, 再通过支撑辊轴承座推动上支 撑辊和上工作辊上下运动, 最终形成工作辊辊缝尺 寸, 得到出口板厚。

弹跳方程描述为

$$
h=S_{0}+\frac{P}{k}
$$

式中 $h$ 一一带钢出口厚度;

$S_{0}$ 一一轧机空载辊缝;

$P$ 一一轧制力;

$k$ 一一轧机纵向刚度。

弹跳方程描述了出口厚度和初始辊缝及轧制 力的关系, 在厚控系统中有着非常重要的作用。空 载辊缝 $S_{0}$ 可以表示为

$$
S_{0}=c-x_{p}-x_{t}-x_{e}+x_{m}
$$

式中 $c$ 一由轧机结构决定的常数;

$x_{P}$ 一伺服液压缸位移;

$x_{t}$ ——轧辊热膨胀量;

$x_{e}$ 一轧辊的偏心量;

$x_{m}$ 一轧辊的磨损量。

把式(2)代入式(1)可得

$$
h=\left(c-x_{p}-x_{t}-x_{e}+x_{m}\right)+\frac{P}{k}
$$

对式(3)两边取增量得

$$
\begin{gathered}
\Delta h=-\Delta x_{P}-\Delta x_{t}-\Delta x_{e}+\Delta x_{m}+\frac{\Delta P}{k} \\
\text { 令 } \Delta x_{r}=\Delta x_{t}+\Delta x_{e}-\Delta x_{m} \text {, 则 } \\
\Delta x_{r}=\frac{\Delta P}{k}-\Delta h-\Delta x_{p}
\end{gathered}
$$

式(5)中轧制压力增量 $\Delta P$, 厚度增量 $\Delta h$, 压下 增量 $\Delta x_{P}$ 均可以由现场检测数据作差得到。 $\Delta x_{r}$ 是 轧制数据的一个综合量, 代表辊系的径向变形。在 较短的时间内, 轧辊磨损以及热变形变化缓慢, $\Delta x_{t} \approx 0, \Delta x_{m} \approx 0$, 则式(1)可简化为

$$
\Delta x_{e}=\frac{\Delta P}{k}-\Delta h-\Delta x_{p}
$$

此式可作为轧辊偏心的在线软测量模型。

\section{2 阶比跟踪与过程数据变换}

对于采用电液伺服自动板厚控制系统的冷带 轧机，轧制力、出口板厚、压下缸位移等信号都 是按照固定的采样周期进行检测和保存的, 但是 
由于测厚仪安装位置和辊缝之间存在一段距离, 厚度信号与轧制力信号和压下量相比存在滞后, 滞后时间与轧制速度有关, 所以要利用式(6)进行 轧辊偏心分析时, 需要将轧制区采集的压力信号、 位移信号进行平移来实现数据匹配。在轧制过程 中, 轧制速度及轧辊的转速按照规程发生变化, 因此厚度信号的滞后时间不是常值, 而是带钢出 口速度的函数。要在时域内实现采集信号的数据 匹配十分困难。同时由于轧制过程中轧制速度变 化, 造成轧辊偏心频率和周期的变化以及信号分 析时的频率混叠。

将阶比跟踪技术应用到车辊偏心信号分析中， 对轧制过程数据进行角域重采样后, 可以解决轧制 速度变化带来的轧辊偏心频率和周期的变化, 而且 厚度信号在角域内滞后量与轧制速度无关, 易于通 过的平移变换实现数据匹配。

\section{3 轧制数据角域重采样算法}

阶比跟踪的关键在于获得相对参考轴的恒定 角增量采样数据, 即实现等角度采样。这在实施上需 要能准确获得阶比采样的时刻(时标)及相应的基准 转速(或频率), 然后应用插值计算, 得到相应采样时 刻的采样值。

冷带轧机一般都需要检测和控制轧辊的转速, 其线速度称为主轧制速度 $v$ 。同时需要监控的还有 入口板带速度 $v_{i}$ 、出口板带速度 $v_{o}$ 。经过时域采样 后, 得到主轧制速度序列 $v\left(t_{i}\right)$ 、出口速度序列 $v_{o}\left(t_{i}\right)$ 、压下位移序列 $x\left(t_{i}\right)$ 、轧制力序列 $p\left(t_{i}\right)$ 、出 口厚度序列 $h\left(t_{i}\right)$ 。

在较小的时间范围内, 可假设轧制速度变化为 匀加速过程, 则轧制速度可表达为二阶拟合方程

$$
v(t)=a t^{2}+b t+c
$$

根据最小二乘法, 拟合误差 $\varepsilon$ 的平方和为

$$
V(a, b, c)=\sum_{k=1}^{K}\left[v\left(t_{k}\right)-v_{k}\right]^{2}
$$

式中, $K$ 为拟合数据点数。

令

$$
\frac{\partial V}{\partial \mathbf{a}}=\frac{\partial V}{\partial b}=\frac{\partial V}{\partial c}=0
$$

求出方程系数 $a 、 b 、 c$, 若设最大分析阶比范 围为 30 , 等角度采样每转采样 60 点。

目前阶比跟踪的主要方法如下：硬件阶比跟踪 法和计算阶比跟踪法。硬件阶比跟踪法需要由转速 脉冲提供对原始信号实现等角增量采样的鉴相信号
(时标), 最大的优点就是不需要添加其他硬件, 用 常规的采集系统结合软件编程就可以实现阶比跟 踪 ${ }^{[6-7]}$ 。在本文提出的方法中并没有直接可供使用的 鉴相信号，故采用计算阶比跟踪法。可用上面得到 的小范围最小二乘拟合主轧制速度曲线 $v(t)$ 来产生 相应的鉴相时标。设各重采样时刻为 $T_{n}, v(t)$ 为瞬 时速度，则有

$$
2 \pi \int_{T_{0}}^{T_{n}} v(t) \mathrm{d} t=n \Delta \theta
$$

式中 $n$ 一一采样时刻序列号;

$\Delta \theta$ 一恒定角速度增量;

$\Delta \theta=\frac{2 \pi}{O_{\max }} ;$

$O_{\text {max }}$ 一最大阶比范围。

将 $v(t)=a t^{2}+b t+c$ 代入式(9)，则

$$
\frac{a T_{n}^{3}}{3}+\frac{b T_{n}^{2}}{2}+c T_{n}-\left(\frac{a T_{0}^{3}}{3}+\frac{b T_{0}^{2}}{2}+c T_{0}\right)=n \Delta \theta
$$

求此方程的有效解，即可求得等角度采样的鉴 相时标 $T_{n}(n=1,2, \cdots, N), N$ 为阶比分析数据长度。

取得角域周期采样时标后, 只要针对各时域序 列在时标 $T_{n}$ 处进行插值计算，即可得到角域周期采 样(重采样)序列值, 插值公式可取 Langrange 线性插 值计算公式

$$
\begin{gathered}
X\left(T_{n}\right)=X\left(t_{i}\right)+\frac{X\left(t_{i+1}\right)-X\left(t_{i}\right)}{t_{i+1}-t_{i}}\left(T_{n}-t_{i}\right) \\
t_{i} \leqslant T_{n} \leqslant t_{i+1}
\end{gathered}
$$

\section{4 厚度信号的角域平移}

由于出口板带厚度信号来自测厚仪, 而测厚仪 需要安装在距辊缝 $L$ 的位置。在同一规程下出口速 度与主轧制速度是正比关系

$$
v_{o}=(1+S) v
$$

式中, $S$ 为前滑系数。

设厚度信号角域滞后 $m$ 个周期, 则

$$
\begin{aligned}
& L=(1+S) m \Delta \theta \\
& m=\left|\frac{L}{(1+S) \Delta \theta}\right|
\end{aligned}
$$

记厚度信号的角域平移后序列为

$$
h^{\prime}\left(T_{n}\right)=h\left(T_{n+m}\right)
$$

为了简便起见, 以下记角域序列 $X\left(T_{n}\right)$ 为 $X(n)$, 经过重采样, 可以得到轧辊偏心角域信号软 测量算法 


$$
\Delta x_{\mathrm{e}}(n)=\frac{\Delta P(n)}{M}-\Delta h_{n}(n+m)-\Delta x_{p}(n)
$$

\section{5 角频域下的偏心信号提取}

在 300 试验轧机轧制过程中, 采集支撑辊 10 转内的数据, 时域偏心信号如图 2 所示, 转速信号 如图 3 所示。将时域偏心信号按照 $\Delta \theta=\pi / 30$ 进行角 域重采样处理, 然后按照式(16)进行综合, 得到角 域偏心数据序列, 如图 4 所示。分别对时域偏心信 号、角域偏心信号进行频谱分析, 如图 5、6 所示, 可看出, 时域偏心信号受转速变化的影响, 频率成 分出现混叠现象, 而角域偏心信号的频率成分清晰, 主要包括 $2 \pi$ 角频率及其倍频成分, 更有利于信号的 分析和提取。图 5 中曲线显示由现场数据综合得到 的偏心信号中仍然包含较强着噪声信号, 需要进一 步的数据处理。

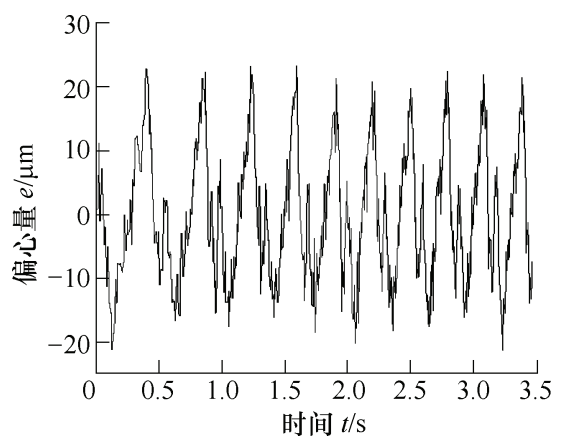

图 2 时域偏心信号

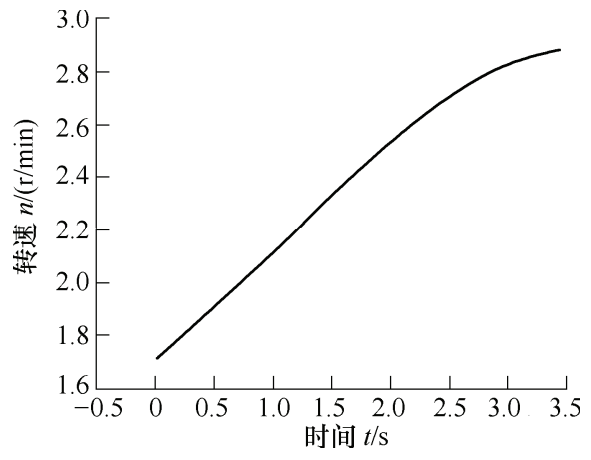

图 3 支撑辊转速信号

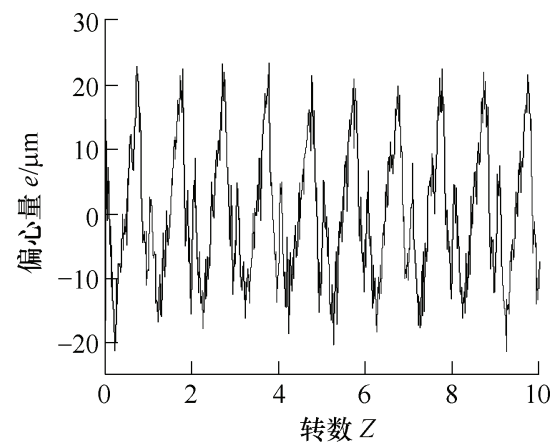

图 4 角域偏心信号

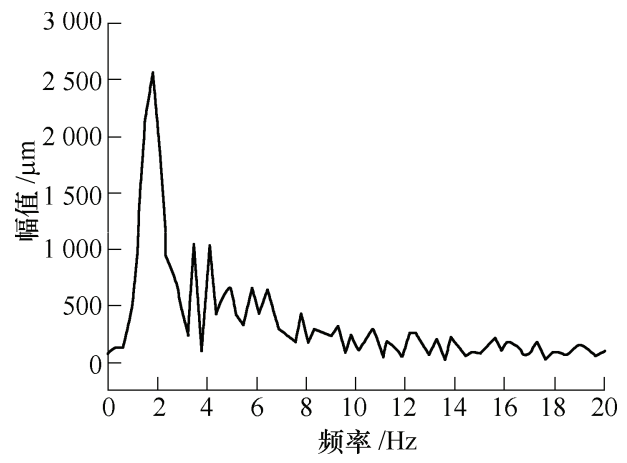

图 5 时域偏心信号的幅频图

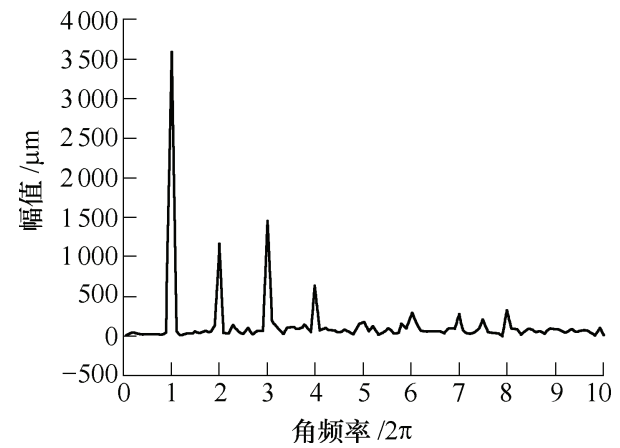

图 6 角域偏心信号的幅频图

对角域偏心数据序列进行小波滤波 ${ }^{[8]}$, 小波函数 选择 sym6, 分解层数为四层, 节点 $(4,0)$ 的频率范围 为 $\left[\begin{array}{ll}0 & 0.1875\end{array}\right]$, 节点 $(4,1)$ 的频率范围为 [1.875 3.75], 节点 $(4,2)$ 的频率范围为 [5.625 7.5], 节点 $(4,3)$ 的 频率范围为 3.75 5.625], 节点 $(4,6)$ 的频率范围为 [7.5 9.375]。滤波后结果如图 7 所示。

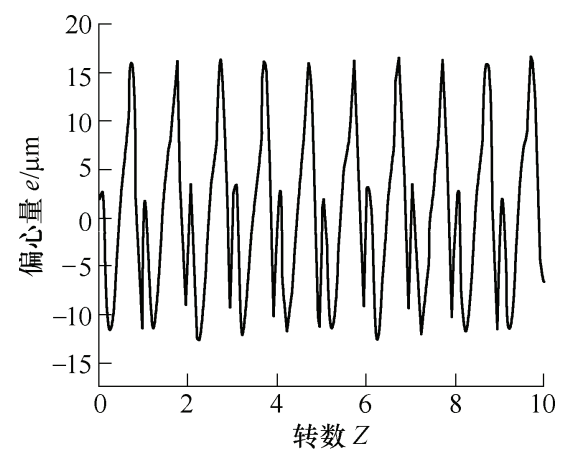

图 7 小波滤波后的偏心信号

为了提高分析所得轧辊偏心波形精度, 轧辊每 转采集 60 个数, 共采集 10 转, 对所采集数据采用 相干时间平均法 ${ }^{[9-11]}$, 求得每周期内各点对应的平 均偏心量

$$
e[n]=\frac{1}{Z} \sum_{z=1}^{Z} x(n+60 z)
$$

式中, $n$ 为轧辊 60 等分转角相位序号; $Z$ 为采集信 号时支承辊的转数, 此处 $Z=10$ 。

最后得到的偏心波形如图 8 所示。图 8 中, 曲 线 1 为由图 7 提取的角域偏心波形, 曲线 2 为在空 载条件下以位置闭环进行压靠试验, 从轧制力信号 


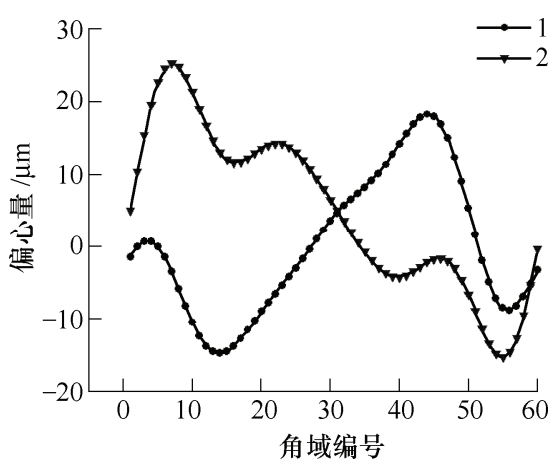

图 8 轧辊偏心波形

中提取的偏心信号。

两曲线但频率成分、幅值范围基本一致，但在 时域上来看, 有较大的差异, 主要是由实际轧制与 压靠试验相比更复杂、辊系间会发生打滑等因素造 成的。

\section{6 结论}

(1) 通过引入阶比跟踪技术, 对轧制过程数据 进行了轧辊旋转等角度采样, 避免了因为轧制速度 变化引起的轧辊偏心频率变化造成的频率混叠 问题。

（2）通过厚度信号角域平移解决了厚度信号滞 后带来的数据不匹配的问题。

(3) 充分利用轧制过程数据分析得到了轧辊偏 心波形, 这种分析方法, 比传统的分析方法更接近 轧制过程的实际情况, 有利于发现并跟踪轧辊偏心 波形的变化, 为轧辊偏心的在线识别和动态补偿奠 定了基础。

\section{参 考 文 献}

[1] 李勇, 刘相华, 王君, 等. 轧辊偏心及其控制问题的分 析与展望 $[J]$. 轧钢, 2006, 23(5): 43-47.

LI Yong, LIU Xianghua, WANG Jun, et al. Analysis and prospect of roll eccentricity and its control[J]. Steel Rolling, 2006, 23(5): 43-47.

[2] 牛满科, 杨卫东, 张丽娟. 轧机偏心补偿控制信息相域 滤波提取方法[J]. 北京科技大学学报, 2011，33(1): 99-104.

NIU Manke, YANG Weidong, ZHANG Lijuan. Phase domain filtering pick up method for roll eccentricity compensation control[J]. Journal of University of Science and Technology Beijing, 2011, 33(1): 99-104.

[3] 郭瑜, 秦树人, 汤宝平, 等. 基于瞬时频率估计的旋转 机械阶比跟踪[J]. 机械工程学报, 2003, 39(3): 32-36 GUO Yu, QIN Shuren, TANG Baoping, et al. Order tracking of rotating machinery based on instantaneous frequency estimation[J]. Chinese Journal of Mechanical Engneering, 2003, 39(3): 32-36.

[4] LI Ning, QIN Shuren, MAO Yongfang, et al. Gabor order tracking filtering technology in rotary machinery[J]. Chinese Journal of Mechanical Engneering, 2010, 23(5): 613-619.

[5] LIU Jingfang, YU Yueqing, HUANG Zhen, et al. General order principle for multi-bennett linkages[J]. Chinese Journal of Mechanical Engineering, 2013, 26(2): 275-281.

[6] 彭富强, 于德介, 武春燕. 基于自适应时变滤波阶比跟 踪的齿轮箱故障诊断 [J]. 机械工程学报, 2012, 48(7): 77-85.

PENG Fuqiang, YU Dejie, WU Chunyan. Self-adaptively time-varying filter based order tracking method and its application in gearbox fault diagnosis[J]. Journal of Mechanical Engineering, 2012, 48(7): 77-85.

[7] 赵晓平, 侯荣涛. 基于 Viterbi 算法的 Gabor 阶比跟 踪技术[J]. 机械工程学报, 2009, 45(11): 247-252.

ZHAO Xiaoping, HOU Rongtao. Gabor order tracking based on Viterbi algorithm[J]. Journal of Mechanical Engineering, 2009, 45(11): 247-252.

[8] 郑建国, 石智, 权豫西. 非平稳信号的小波包阈值去噪 方法[J]. 信息技术, 2007(3)：16-17.

ZHENG Jianguo, SHI Zhi, QUAN Yuxi. Wavelet packet threshold approach for denoising non-stationary signal[J]. Information Technology, 2007(3): 16-17.

[9] 陈志菲, 孙进才，牛奕龙. 基于相干平均的正弦信号频 率估计[J]. 西北工业大学学报, 2009, 27(3): 387-390.

CHEN Zhifei, SUN Jincai, NIU Yilong. A better method of frequency estimation for sinusoidal signals using coherent average[J]. Journal of Northwestern Polytechnical University, 2009, 27(3): 387-390.

[10] CHRISTOPHER T A, SHADAB N M, TORRY L A. Suppressing coherent noise in radar applications with long dwell times[J]. IEEE Geoscience and Remote Sensing Letters, 2005, 2(3): 284-286.

[11] 李伯群, 孙一康, 傅剑, 等. 基于相位补偿的轧辊偏心 控制[J]. 钢铁研究学报, 2006, 18(6): 55-58.

LI Boqun, SUN Yikang, FU Jian, et al. Control of roller eccentricity based on phasic compensation strategy[J]. Journal of Iron and Steel Research, 2006, 18(6): 55-58.

作者简介: 刘涛(通信作者), 男, 1973 年出生, 博士, 副教授。主要研 究方向为治金机械及机电液综合控制系统。

E-mail: liutao@ysu.edu.cn 